\title{
A pesquisa clínica no Brasil
}

\author{
Clinical research in Brazil
}

Marco Antônio Zago 1

\footnotetext{
1 Faculdade de Medicina de Ribeirão Preto, USP. Hemocentro. Rua Tenente Catão Roxo, 2501. 14051-140 Ribeirão Preto SP marazago@usp.br
}

\begin{abstract}
Strengthening clinical research in Brazil involves the creation, expansion, or consolidation of new centers, and especially the recovery of university hospitals and a stronger link between teaching and research. Interaction between biomedical and basic research is indispensable to generate national knowledge on specific issues and to structure the health technology production sector. Changes in the country's demographic and epidemiological profile have brought cardiovascular diseases, neoplasms, psychiatric diseases, diseases associated with urbanization, accidents/violence, and diseases of ageing to the list of health research priorities. Brazil still displays important endemics in its epidemiological profile: dengue, malaria, schistosomiasis, Hansen's disease, and viral hepatites, among others. Consolidation of research in cellular and molecular biology applied to medicine is indispensable for medical practice in the country to remain up-to-date and competitive, as well as to allow interactions with the productive sector and the implementation of a national biotechnology complex.
\end{abstract}

Key words Clinical research, Scientific and technological development, Research agenda
Resumo O fortalecimento da pesquisa clínica no Brasil implica a criação, expansão ou consolidação de novos centros, em especial na recuperação dos hospitais universitários e do fortalecimento do vínculo entre ensino e pesquisa. A interação entre pesquisa biomédica e básica é indispensável para geração de conhecimento nacional em questões específicas e para estruturar o setor produtivo de tecnologia ligada à saúde. As mudanças do perfil demográfico e epidemiológico do país trouxeram para a lista de prioridades de pesquisa em saúde doenças cardiovasculares, neoplasias, doenças mentais, doenças associadas a urbanização, acidentes e violência, e doenças do envelhecimento. O Brasil ainda apresenta endemias importantes no seu quadro epidemiológico: dengue, malária, esquistossomose, hanseníase e hepatites virais, entre outras. A consolidação da pesquisa em biologia celular e molecular aplicada à medicina é indispensável para que a prática médica no país se mantenha atualizada e competitiva, e para permitir interações com o setor produtivo e implantação de um parque biotecnológico nacional. Palavras-chave Pesquisa clinica, Desenvolvimento científico e tecnológico, Agenda de pesquisa 


\section{Introdução}

O tema comporta duas abordagens distintas, mas não antagônicas, empregadas em recente revisão no contexto da Conferência Nacional de Ciência, Tecnologia e Inovação (Zago et al., 2002). Uma primeira maneira de tratar o assunto é fazer uma análise da situação atual da pesquisa clínica no Brasil, procurando projetála no cenário global e buscando identificar os seus pontos fortes e as debilidades, no sentido de propor correções. Por outro lado, partindo da situação epidemiológica, de saúde pública e demográfica do país, e considerando o cenário global da medicina, é possível identificar uma agenda de temas e ações que buscam atender a dois objetivos centrais: a melhoria da qualidade de vida da população brasileira, e o fortalecimento da medicina e da indústria biotecnológica e farmacêutica do país. Mais polêmica, exige um juízo crítico e análise de valor, que aumentam o seu interesse, é pois a proposta que adotarei neste artigo.

Essa segunda abordagem tem um pressuposto conceptual e prático: a pesquisa médica não se realiza de maneira absolutamente independente da situação prática do exercício da medicina e das condições de saúde da população. Em seu limite, uma visão como esta pode levar a restrições indesejáveis à pesquisa clínica e biomédica, prejudicando a liberdade de ação dos cientistas, inibindo sua capacidade criativa e culminando com um tipo de direcionamento científico que já se demonstrou desastroso em vários cenários. No entanto, é inevitável reconhecer que a pesquisa, em especial a pesquisa biomédica e clínica, não se faz por diletantismo nem apenas em nome de um valor abstrato do conhecimento científico. Há que haver um equilíbrio. Ao mesmo tempo em que é necessário preservar a liberdade de ação do cientista na escolha de temas e objetos de pesquisa, protegendo o exercício da pesquisa que não visa a resultados imediatamente aplicáveis, o custo das atividades científicas e o papel central do conhecimento científico e tecnológico como fator de independência nacional exigem uma política de longo prazo para orientar os grandes investimentos públicos na área, focalizada em problemas cuja solução pode se refletir em aumento do bem-estar da população. Para ser efetiva essa política deve também contemplar a formação de recursos humanos no setor, em especial de graduação e pós-graduação em medicina, e os próprios sistemas de gestão e de avaliação dos investimentos para o setor de ciência e tecnologia.

\section{Interação da pesquisa clínica com saúde pública e biotecnologia}

De uma maneira ampla, "pesquisa em saúde" inclui hoje três componentes complementares e altamente interdependentes, a saber: a) a pesquisa clínica (incluindo todas as variantes em que o foco é o paciente (real ou potencial), isolado ou coletivamente); b) a pesquisa dos sistemas de saúde (incluindo saúde coletiva, organização e gestão, epidemiologia); e c) as ciências básicas e biotecnologia (consideradas em seus aspectos que podem resultar em aplicações para diagnóstico ou tratamento, em grande parte contemplados sob o título de pesquisas biomédicas). Neste artigo não vamos considerar os importantes componentes representados pela pesquisa focalizada nos sistemas de saúde ou em ciências básicas e biotecnologia, mas entendemos que, em conjunto com a pesquisa clínica, devem contribuir fortemente para influenciar as políticas públicas e resolver problemas atuais da população brasileira, tais como:

1. demanda crescente por novos serviços, resultante do envelhecimento da população e da rápida inovação da tecnologia médica voltada ao diagnóstico, ao tratamento e à readapatação de pacientes com doenças crônicas;

2. persistência de desigualdades sociais que se refletem na qualidade de vida e da saúde, contribuindo para prevalência de doenças infecciosas e distúrbios derivados da miséria ou da falta de saneamento;

3. necessidade de otimizar o uso dos recursos disponíveis para o setor de saúde;

4. crescente importância dos agravos resultantes ou associados à urbanização e industrialização, tais como violência, acidentes, alcoolismo e adição a drogas;

5. necessidade de criar uma indústria farmacêutica, hospitalar, laboratorial e biotecnológica própria, com independência tecnológica, capaz de atender às nossas necessidades e participar do esforço de exportação e fortalecimento econômico do país.

\section{A formação de recursos humanos}

Apenas 10\%-20\% dos cerca de 9.500 médicos formados anualmente no país têm contato com 
o sistema de pesquisa médica. Considerandose o papel formativo que a pesquisa e o método científico têm sobre a educação do médico, conclui-se que uma parcela significativa dos médicos brasileiros é formada à margem desse sistema, não estando preparada para liderar ou pelo menos acompanhar e absorver as inovações no setor de saúde, que são altamente vinculadas ao desenvolvimento científico. Neste aspecto, a inovação e o progresso científico e tecnológico são extremamente dependentes da melhora qualitativa do ensino de medicina, que não pode ser desvinculado da formação científica e geração de conhecimento. Essa etapa é crítica não apenas para a formação dos futuros pesquisdores como também como parte do treinamento do médico para a compreensão e aplicação de resultados de pesquisas realizadas por outros como para a absorção e utilização educada dos progressos da medicina após a sua formação.

A pós-graduação vincula-se solidamente à pesquisa, pois exige uma massa de orientadores com título de doutor, e a titulação de cada egresso somente pode ser alcançada pela realização de um trabalho de pesquisa. Mesmo assim, em muitos programas ocorre uma grande influência da formação profissionalizante e especialização médica, contribuindo para desviálos de seus focos básicos: a produção de pesquisa e qualificação de pesquisadores. O panorama neste caso é também misto: revela um conjunto de instituições que estão executando uma política de estímulo à pesquisa e de formação de pesquisadores, enquanto mais da metade dos cursos de pós-graduação na área médica estão longe de cumprir sua função central, assumindo, em muitos casos, uma formação profissionalizante. A manutenção de um sistema de avaliação de desempenho da pós-graduação firme e consistente por parte da Capes, combinada com uma política de restrição aos programas que não satisfazem às exigências mínimas e de incentivo àqueles que procuram atender às metas de incremento de pesquisa, pode ter um grande impacto no aumento da eficiência científica da área médica.

\section{Os centros de pesquisa médica}

A pesquisa médica mais expressiva realiza-se no Brasil predominantemente em cerca de duas dezenas de centros, em sua grande maioria de natureza pública, fortemente concentra- dos no Sul e Sudeste, em especial universidades federais e estaduais e algumas instituições isoladas. Sem particularizar os detalhes, esta observação conduz a duas conclusões importantes: a) necessidade de reduzir o desequilíbrio regional, e b) a existência de uma ampla rede de hospitais universitários não utilizados adequadamente como sede de pesquisa médica.

Uma política de recuperação dos hospitais universitários parece uma alternativa viável para ampliar e descentralizar a atual rede de pesquisa em saúde, desde que associada à qualificação de recursos humanos, em especial à formação de pesquisadores, e a uma clara vinculação de recursos a projetos de pesquisa, e não a finalidades exclusivamente assistenciais.

\section{Qual deve ser o foco da pesquisa clínica?}

A avaliação das condições de pesquisa médica e, em especial, uma projeção de prioridades, exige um claro discernimento entre o sistema de saúde e o de bem-estar social. Apesar das interações entre eles, grandes problemas de saúde resultantes de falta de saneamento, de educação ou resultantes da miséria e do desemprego, não podem ser solucionados com a pesquisa médica ou social, mas exigem ações governamentais ou institucionais, predominantemente (mas não exclusivamente) públicas. Por isso, uma análise crítica dos temas prioritários de pesquisa médica e biomédica de interesse para o país não pode incluir tópicos que, apesar de seu elevado apelo emocional, não podem de fato produzir conhecimento novo capaz de contribuir para a solução dos problemas de saúde resultantes da miséria. A pesquisa visa criar novo conhecimento ou reorganizar o conhecimento existente pela análise crítica.

Talvez o elemento central no planejamento de desenvolvimento científico na área médica deva ser o de reforçar a necessidade de adquirir nossa própria experiência: o Brasil precisa obter seus próprios dados, formular políticas próprias e resolver problemas específicos do país. Componente adicional a ser considerado é o elevado custo dos procedimentos médicos e de assistência à saúde, incluindo o diagnóstico, medicamentos, equipamentos médicos, hospitalares e laboratoriais, entre outros. Parte considerável e crescente desses recursos são direta ou indiretamente transferidos para o exterior. O desenvolvimento de tecnologia nacional, capaz de competir com a importada ou substituí- 
la, deve também constituir uma prioridade, mesmo para temas que não são prioritários pela sua importância epidemiológica no país.

\section{O perfil epidemiológico da população brasileira}

O perfil epidemiológico coloca o país claramente na categoria que o Global Forum for Health Research classifica como dupla carga (<http://www.globalforumhealth.org/>). Em amplas regiões do país, que incluem os grandes centros urbanos e a maior parte das regiões Sul e Sudeste, predomina o perfil epidemiológico próprio de países com renda elevada (predomínio de doenças não-transmissíveis); no entanto, em outras regiões, mas também convivendo nos grandes centros urbanos mais desenvolvidos, há contingentes de populações com perfil epidemiológico próprio de países de renda baixa, em que as doenças transmissíveis, as condições perinatais e maternas e aquelas derivadas de desnutrição têm uma posição proeminente. A complexidade do quadro é maior porque não há uma separação exclusivamente geográfica dos dois perfis epidemiológicos, mas as populações afetadas pelos dois padrões têm uma distribuição complexa, que é ao mesmo tempo determinada pela geografia e vinculada ao perfil socioeconômico. Finalmente, os agravos à saúde derivados do terceiro perfil epidemiológico afetam indiferentemente os dois grupos anteriores: alcoolismo, adição a drogas, violência urbana e rural, acidentes e poluição. De um modo geral, a avaliação com base nas causas de mortalidade mostra um perfil em que predominam as doenças crônico-degenerativas e não-transmissíveis, o que representa uma sensível mudança em relação às décadas anteriores (Tabela 1).

As doenças do aparelho circulatório representam a principal causa de óbitos no país (32\%) e em todas as regiões, seguidas pelas causas externas (15\%), neoplasias (15\%) e doenças do aparelho respiratório (11\%). Entre 1980 e 1999, observaram-se aumento na proporção destas causas e diminuição das doenças infecciosas e parasitárias - de $9 \%$ para $6 \%$ - e das afecções originadas no período perinatal - de $7 \%$ para 5\% (<http://portal.saude.gov.br/saude/>).

Essa modificação do perfil epidemiológico da população brasileira tem várias causas, entre elas o impressionante aumento da proporção de idosos. O desenvolvimento social e eco-
Tabela 1

Principais causas de morte no Brasil, em 1999.

\begin{tabular}{lc}
\hline Grupo de doenças & Porcentagem do total \\
\hline Cardiocirculatórias & $32,3 \%$ \\
Causas externas & $14,7 \%$ \\
Neoplasias & $14,5 \%$ \\
Doenças respiratórias & $11,2 \%$ \\
Doenças infecto-parasitárias & $5,8 \%$ \\
Outras causas & $21,5 \%$ \\
\hline
\end{tabular}

Fonte: Ministério da Saúde

nômico, a melhora do saneamento básico, da alimentação e da educação, associados à evolução da medicina, têm elevado acentuadamente a expectativa média de vida. No Brasil, a expectativa de vida ao nascer sofreu um incremento significativo, saltando de 33,7 anos em 1900 para 63,5 anos em 1980 (Ramos LR et al. 1987), ao qual se associa um aumento, na população geral, do número de pessoas com mais de 80 anos, constituindo a denominada "quarta idade”. De 1900 a 2025 a população brasileira terá sido multiplicada por cinco e o segmento de pessoas com mais de 60 anos terá o seu número multiplicado por 15 , fazendo com que o Brasil passe de 16o (em 1950) para o 6o lugar mundial (em 2025) em número absoluto de pessoas com 60 anos ou mais, quando um em cada sete brasileiros fará parte do contingente idoso da população e teremos uma expectativa de vida ao nascer de aproximadamente 73 anos.

Desnecessário enfatizar que esta impressionante e rápida mudança do perfil populacional não só tende a aumentar a importância das doenças cardiovasculares, pulmonares e neoplásicas como causa de mortalidade e morbidade da população, mas traz para o topo da lista de prioridades questões próprias dos idosos, como a convivência com deficiências, diabete, osteoporose e fraturas, Alzheimer e demências. Como conseqüência, o padrão de doenças que provocam internações e morte nos idosos distingue-se daquele observado na população geral (tabela 2).

Embora não apareçam nas tabelas de mortalidade, as doenças mentais ocupam um lugar muito importante pela sua morbidade e prejuízo econômico e repercussão social. Os distúrbios mais relevantes nesta categoria incluem ansiedade, depressão e dependência do álcool. 
Tabela 2

A morbidade hospitalar (\% do total de internações) e mortalidade proporcional (\% dos óbitos devidos àquela causa) por grupos de doenças, na faixa etária superior aos 60 anos, no ano de 1999.

\begin{tabular}{lcc}
\hline Grupo de doenças & Internações & Mortalidade \\
\hline Doenças do aparelho respiratório & $21,42 \%$ & $15,45 \%$ \\
Doenças do aparelho circulatório & $28,76 \%$ & $45,51 \%$ \\
Doenças do aparelho digestivo & $9,95 \%$ & \\
Doenças do aparelho geniturinário & $6,05 \%$ & $3,54 \%$ \\
Doenças infecciosas e parasitárias & $5,80 \%$ & $16,57 \%$ \\
Neoplasias & $4,43 \%$ & $3,20 \%$ \\
Lesões e envenenamentos & $4,16 \%$ & $15,73 \%$ \\
Transtornos mentais & $2,05 \%$ & $17,34 \%$ \\
Demais causas & & \\
\hline
\end{tabular}

Fonte: Datasus.

\section{Grandes temas de pesquisa clínica}

Consoante o exposto, alguns temas devem necessariamente fazer parte de uma agenda de prioridades para as pesquisas em medicina clínica no Brasil. Esta não é uma lista clássica de prioridades para pesquisa que alguns organismos ou grupos desastradamente procuram elaborar. Essas listagens padecem de dois defeitos: a) para serem amplas e inclusivas resultam muitas vezes em enumerações infindáveis que incluem tudo ou quase tudo, perdendo, pois, qualquer sentido prático; ou b) enumeram algumas doenças ou condições segundo a visão de um alguns, criando mal-estar pela não-inclusão deste ou daquele tema.

Acredito que a) o perfil epidemiológico da população brasileira, b) as projeções de evolução demográfica, c) a necessidade do desenvolvimento da indústria farmacêutica e biotecnológica no país projetam um cenário de temas que obrigatoriamente se consolidarão, sem necessidade de dirigismo.

\section{Câncer}

O câncer constitui a terceira causa de morte no país, e a tendência é aumentar sua relevância à medida que aumenta a expectativa de vida da população e há uma redução de mortalidade por doenças transmissíveis. Para o ano de 2001 projetou-se a ocorrência de cerca de 305.330 casos novos de câncer no país e cerca de 117.500 mortes causadas pelas diferentes formas da doença, predominando os cânceres do sistema respiratório, estômago, mama, próstata e coloretal (Instituto Nacional do Câncer, 2001).
A importância crescente deste grupo de doenças não tem sido acompanhada de aumento proporcional do esforço de investigação e de investimentos para pesquisa, apesar de ter ocorrido um sensível progresso nos últimos anos, revelado por:

- Aumento da competência profissional médica para diagnóstico e tratamento de câncer, concentrada ainda em alguns hospitais e centros universitários, embora haja uma tendência a se difundirem. Esta proficiência envolve principalmente a área cirúrgica, a manipulação de quimioterápicos e suporte ao paciente com câncer e imunodeprimido. Os métodos diagnósticos mais precisos e invasivos são ainda de uso restrito, pela carência de pessoal treinado e pelos altos custos envolvidos, assim como abordagens especiais de tratamento. Por exemplo, ainda há um número restrito de centros no país que realizam rotineiramente o transplante alogênico de medula óssea, uma forma bem-estabelecida de tratamento de neoplasias hematológicas.

- Aumento dos artigos e dos projetos de pesquisa na área de oncologia, incluindo numerosos projetos que tratam de aspectos básicos, moleculares ou de aplicação de conhecimentos básicos à área clínica, e não apenas testes de medicamentos. Um expressivo esforço recente de pesquisa em câncer no país envolveu uma iniciativa conjunta (ação provocada e não de demanda) da Fapesp e do Ludwig Institute for Cancer Research, e representa um excelente exemplo da associação da pesquisa médica com pesquisa básica para geração de conhecimento genuinamente nacional em questões específicas (Bonalume Neto, 1999; Souza et al., 2000; Dias Neto et al., 2000). 
- Estabelecimento e modernização de hospitais e institutos dedicados ao tratamento e estudo do câncer.

Uma agenda incompleta de tópicos que devem focalizar a atenção da pesquisa de câncer no país nos próximos anos inclui: a) variações da expressão gênica em tumores, relação de alterações gênicas específicas com a suscetibilidade à doença, sua gênese, evolução e resposta terapêutica; b) associação de mecanismos celulares e variações histopatológicas com a evolução e resposta ao tratamento; c) desenvolvimento de novas abordagens terapêuticas com base nos conhecimentos de biologia celular e molecular; d) identificação de fatores de risco e fatores ambientais associados aos diversos cânceres e propostas de estratégias de prevenção; e) estabelecimento e manutenção de registros de base populacional; f) desenvolvimento e aperfeiçoamento de estratégias de tratamentos quimioterápicos, radioterápicos, com agentes biológicos ou transplantes, em especial por meio de testes clínicos em grupos de colaboração; g) desenvolvimento de estratégias de tratamento para reduzir as mutilações, e de abordagens educativas e de apoio para recuperação dos pacientes; $h$ ) fatores determinantes de qualidade de vida, e manutenção da autonomia e capacidade funcional.

\section{Geriatria e gerontologia}

A identificação de prioridade deve levar em conta o peso dos diferentes problemas de saúde entre idosos e a situação atual da pesquisa em geriatria no país. Os informes epidemiológicos do SUS apontam as doenças do aparelho circulatório, as neoplasias e as doenças do aparelho respiratório como as principais causas de óbito e de hospitalização após os 60 anos de idade.

A elevada freqüência de doenças crônicodegenerativas em idosos aumenta o uso de recursos hospitalares. O aumento da utilização de recursos de saúde hospitalares e ambulatoriais, com implicações econômicas sérias e evidentes (já demonstradas em outros países), eleva o envelhecimento populacional à condição de grave problema de saúde pública. A abordagem correta desses pacientes só pode ser atingida pelo treinamento adequado de profissionais da área de saúde, e pelo estímulo à pesquisa em temas relacionados com o envelhecimento, desde a compreensão de suas bases biológicas e moleculares (como as demências, as alterações do metabolismo energético, protéico e ósseo em idosos, e o câncer), como de temas diretamente relacionados com a assistência à saúde, a manutenção da qualidade de vida, da autonomia e de funções da vida diária.

As doenças neurodegenerativas, incluindo a doença de Alzheimer e outras demências, apesar de não sobressaírem nas estatísticas de internações hospitalares, têm prevalência importante nesta faixa etária, estimada de $5 \%$ aos 65 anos e duplicada a cada cinco anos, após essa idade. A pesquisa de saúde deve ainda focalizar as grandes síndromes geriátricas como: incontinência urinária, quedas, iatrogenia, instabilidade homeostática, estado confusional agudo, e alterações metabólicas como diabete, desnutrição e osteoporose.

A expansão e a consolidação da pesquisa em geriatria no país dependerá de desenvolvimento de duas vertentes: a) ensino e treinamento, em todos os níveis, como a inclusão da geriatria nos currículos de medicina, expansão da residência médica e dos programas de pósgraduação; b) ação positiva dos órgãos financiadores no sentido de estimular a pesquisa em geriatria e gerontologia, em especial a pesquisa fundamental e aquele que pode dar resultados práticos para a atenção à saúde do idoso.

Nessa perspectiva, podem ser apontados alguns tópicos especiais de pesquisa para o desenvolvimento da gerontologia no país: a) mecanismos biomoleculares do envelhecimento, em especial sua compreensão do ponto de vista de biologia celular, molecular e bioquímico; b) metabolismo energético, protéico e ósseo em idosos; c) biologia molecular, bioquímica e fisiologia das demências; d) estudos em doenças cardiovasculares, especialmente aqueles relacionados com a aterosclerose, a insuficiência do miocárdio e a hipertensão; e) bases moleculares e tratamento do câncer; f) índices e fatores determinantes de qualidade de vida; g) manutenção da autonomia e capacidade funcional; h) instrumentos e escalas de avaliação cognitiva, funcional, do humor e de outras esferas.

\section{Saúde mental}

A prevalência aproximada de transtornos mentais da população adulta no Brasil é da ordem de $30 \%$ no período de um ano enquanto que cerca de $20 \%$ da população adulta demanda algum tipo de atenção em saúde mental num período de 12 meses (Almeida-Filho $\mathrm{N}$ et al., 1992; 1997). Entre as mulheres são mais comuns os transtornos de ansiedade (9,0\%), trans- 
tornos somatoformes $(3,0 \%)$ e transtornos depressivos $(2,6 \%)$, enquanto que na população masculina a dependência ao álcool aparece como o problema mais importante $(8 \%)$, seguindo-se os transtornos de ansiedade (4,3\%) (Mari, 1987). Os transtornos psiquiátricos na comunidade são mais freqüentes na população feminina, aumentam com a idade e apontam para um excesso no estrato de baixa renda (Mari, 1992).

Tomando-se como base o cálculo de YLDs (Years Lost for Disabilities), que estima o período que um determinado indivíduo vive com uma determinada incapacitação, chega-se ao diagnóstico da magnitude do efeito que os transtornos mentais exercem na saúde geral da população. Entre as dez condições de maior incapacidade no mundo todo (estimadas pelos YLDs), cinco são devidas a transtornos mentais: depressão, dependência ao álcool, esquizofrenia, transtorno afetivo bipolar e transtorno obsessivo compulsivo (Murray \& Lopez, 1996). $\mathrm{O}$ aumento da longevidade e a melhora dos indicadores de saúde, das últimas décadas, colocaram os transtornos mentais entre as cinco condições mais importantes de saúde pública no Brasil (levando-se em conta os custos diretos e aposentadorias por invalidez), aproximando-os do câncer, das doenças cardiovasculares e das doenças infectocontagiosas.

A pesquisa nesta área sofreu uma forte influência do aprimoramento do processo de avaliação da Capes, que reforçou sua importância no contexto dos cursos de pós-graduação (Mari \& Zago, 2002). Houve um crescimento importante na produção de artigos científicos nos últimos anos, mas que ainda não acompanhou o crescimento de outras áreas da saúde. Este aprimoramento da avaliação dos programas de pós-graduação deveu-se principalmente à aceitação da relevância da produção intelectual, aferida pela qualidade dos veículos científicos, como elemento central de julgamento, desestimulando orientadores que não apresentavam familiaridade com o método científico e produziam predominantemente livros e artigos em revistas locais não arbitradas por especialistas externos.

A dependência ao álcool surge como a condição mais importante de saúde mental no Brasil. Cerca de $8 \%$ da população adulta masculina necessita de algum cuidado médico face ao uso nocivo de álcool, no período de um ano, sendo o álcool responsável por aproximadamente $40 \%-50 \%$ dos casos novos internados em hospitais psiquiátricos, que pode não ser o melhor local de tratamento dessa população. Há uma carência de estudos de custo efetividade para comparar o tratamento hospitalar tradicional com o custo do tratamento em unidades de desintoxicação breve nos hospitais gerais. Como resultado, tanto as pesquisas das bases biológicas como as relacionadas com o cuidado do dependente devem ser estimuladas.

A mudança de perfil demográfico, com predomínio da população urbana em relação à rural, pode estar associada ao excesso de transtornos psiquiátricos menores (principalmente estados de ansiedade). Esta mudança migratória resultou na acentuação de condições habitacionais precárias, dificuldade de inserção ocupacional, falta de lazer, e a violência decorrente da desigualdade social, e recomenda o desenvolvimento ou intensificação de pesquisas relacionadas à saúde mental em áreas urbanas carentes. Paralelamente, o aumento da longevidade e redução das taxas de fertilidade resulta no incremento dos transtornos mentais da terceira idade (depressão e estados demenciais entre outros).

\section{Doenças cardiovasculares}

Representam a primeira causa de morte em todas as regiões do país, essencialmente em virtude de dois componentes, a hipertensão arterial e suas conseqüências e a aterosclerose em suas várias formas. A escassez de dados próprios relacionados a fatores de risco e fatores prognósticos, a grande diversidade regional que se reflete em hábitos alimentares, estilos de vida e padrões de assistência médica, e uma grande diversidade de contribuições étnicas são alguns dos elementos que tornam mais complexas as pesquisas nesta área em nosso país.

Algumas áreas, indicadas a seguir, são de interesse especial em cardiologia no Brasil, em vista da alta incidência de casos, de sua gravidade quanto a morbi-mortalidade e dos custos que acarretam.

Aterosclerose: compreendendo as formas coronária, cerebral e vascular periférica. Alguns tópicos podem ser citados especificamente: a) fatores de risco tradicionais e novos (homocisteína, HDL baixo, síndrome plurimetabólica); b) mecanismos de instabilização de placas; c) detecção não-invasiva da doença; d) viabilidade miocárdica; e) marcadores genéticos de aterosclerose; f) preditores evolutivos quanto às várias formas de apresentação da doença; g) in- 
fluência de dietas, ação de plantas tropicais através de flavonóides; h) disfunção endotelial.

Hipertensão arterial: doença complexa, multifatorial, que resulta do desequilíbrio de múltiplos sistemas, cada um deles como uma regulação complexa, conseqüência da interação de genes e fatores ambientais. Conhecer cada um dos componentes de cada sistema, identificar os genes, conhecer a sua regulação, estabelecer a correlação entre marcadores genéticos e funcionais, e não menos importante, estudar a importância relativa de cada um dos sistemas e saber como eles funcionam integradamente, é o grande campo de pesquisa para as próximas décadas na área da hipertensão e das doenças cardiovasculares. Alguns tópicos poderiam ser citados: a) mapeamento dos genes da hipertensão; b) aspectos moleculares da regulação do sistema renina-angiotensina; c) análise do componente neurogênico; d) análise do sistema óxido nítrico/endotélio; e) papel e formato do treinamento físico.

Arritmias: vias finais comuns que levam à morte em várias situações clínicas, como insuficiência coronária e insuficiência cardíaca congestiva, na qual as mortes súbitas, por arritmias, representam aproximadamente $35 \%$ da mortalidade. Arritmias supraventriculares e ventriculares representam grande número de casos e implicam enormes custos para diagnóstico e tratamentos. Esses tratamentos necessitam de análise quanto à eficiência e custos. Tópicos gerais de interesse incluem: a) mecanismos fisiopatológicos; b) drogas; c) desfibriladores; d) marca-passos; e) ablação; f) estudo da fisiopatologia de arritmias em doença de Chagas e outras cardiomiopatias, e insuficiência coronária; g) novas técnicas para tratamento de arritmias de alto risco: comparação entre drogas, ablação e desfibriladores.

\section{Doenças infecciosas e condições prevalentes na infância}

A despeito do reconhecido progresso da situação de saúde do país, que se expressa, entre outros indicadores, pela queda na mortalidade infantil e pelo controle das principais doenças imunopreviníveis, o quadro epidemiológico brasileiro ainda comporta endemias importantes: dengue, malária, esquistossomose, doença de Chagas, filariose, hanseníase, tuberculose, HPV e hepatites virais, entre outras. Ele está incluído nos chamados "países endêmicos para doenças tropicais” pela Organização Mundial de Saúde (Buss, 2002). A dengue, por exemplo, representa a mais importante arbovirose do mundo e entre as doenças re-emergentes é a que constitui o mais importante problema de saúde pública (Tauil, 2002).

Com tendência constante de queda, a mortalidade infantil atingiu, em 1999, 31,8 óbitos por mil nascidos vivos, com maior participação do componente neonatal (20,1 por mil). Apesar do declínio em todas as regiões, os valores médios ainda são elevados, sendo maiores no Nordeste $(52,4)$ e no Norte $(33,9)$. As menores taxas se encontram nos Estados das regiões Sul e Sudeste, com destaque para o Rio Grande do Sul com 15,1 óbitos por mil nascidos vivos (<http://portal.saude.gov.br/saude/>). Estes dados apontam para a importância crescente da pesquisa relacionada às causas não-infecciosas e nutricionais de mortalidade e morbidade na infância, entre as quais as doenças geneticamente determinadas. Destas, a mais importante é a anemia falciforme. Com prevalência média de heterozigotos de cerca de $2 \%$ $3 \%$ na população geral, é a doença monogênica mais comum do Brasil (Zago et al., 1983; Zago \& Costa, 1985). Embora predomine ainda entre as afro-descendentes, a miscegenação crescente vai fazendo dela uma doença sem vínculo claro com a etnia. A gravidade da doença, que produz morte súbita na infância, numerosas complicações infecciosas, acidente vascular cerebral e reduz signficativamente a expectativa de vida, exige programas de atendimento especial e detecção precoce, incluindo o screening neonatal (Zago, 2001). Além disso, necessita de pesquisas focalizadas na evolução clínica e peculiaridades da doença em nosso ambiente.

A mortalidade proporcional por doença diarréica aguda e por infecção respiratória aguda, em menores de cinco anos, mostra queda no período 1994-1999, em todas as regiões, e alcançou, em 1999, 5,5\% e 6,4\%, respectivamente. A proporção de mortes por doença diarréica aguda é elevada no Nordeste (10\%) e baixa no Sudeste $(2,7 \%)$ - valores altos geralmente estão associados a condições insatisfatórias de saneamento e de atenção à saúde da criança. A mortalidade por infecção respiratória aguda apresenta menor heterogeneidade entre as regiões, com percentuais acima da média nacional para o Norte e o Sudeste (cerca de 7\%).

Para a maioria das doenças infecciosas são bem conhecidos a etiologia, os mecanismos de transmissão e o espectro de manifestações clínicas. Os principais tópicos de pesquisa, que 
podem concorrer para soluções práticas, envolvem a identificação de fatores que contribuem para determinar a gravidade do quadro clínico e sua evolução, métodos diagnósticos mais simples, rápidos e seguros, e novas abordagens terapêuticas e de profilaxia. Neste contexto, o desenvolvimento de vacinas constitui um ponto central, de importância dupla, pois as vacinas representam uma das mais eficientes e menos dispendiosas formas de controle de doenças infecciosas, e em segundo lugar porque existe no país competência para planejamento e produção. De fato, dois centros têm tradição e capacidade instalada para produção de vacinas (Instituto Butantã - <http://www.butantan.gov.br $>$, e a Fundação Oswaldo Cruz por meio de BioManguinhos $-<$ http://www.bio.fiocruz.br $>$ ), e numerosas iniciativas de pesquisadores brasileiros inserem-se neste tema, que deve constituir um tópico prioritário na agenda de pesquisa em saúde.

Duas outras questões vêm assumindo dimensões bastante significativas: as infecções em imunodeprimidos e infecções hospitalares. Em ambos os casos necessitamos adquirir nossa própria experiência nacional e regional, pois vários de seus aspectos, como agentes etiológicos mais comuns, variações nas manifestações clínicas, suscetibilidade aos antibióticos e quimioterápicos, e hábitos da equipe de saúde são muito dependentes de fatores locais, como variabilidade genética da população, condições de atendimento hospitalar, condições socioeconômicas e culturais, treinamento da equipe de saúde, entre outros. O crescimento da importância desses temas liga-se aos outros fatores já citados, como crescimento da população de idosos, aumento da incidência de neoplasias, aumento dos tratamentos altamente invasivos em unidades de terapia intensiva e transplantes, e a infecção por HIV e sua conseqüente imunodepressão.

Finalmente, um tópico que merece atenção particular, vinculado às doenças infecciosas, é a resistência às drogas, em especial da tuberculose e malária.

\section{Biologia molecular e celular}

À medida que a revolução causada pela nova genética (Weatheral, 1985) transferiu-se das bancadas da investigação básica para as aplicações clínicas, inicialmente no diagnóstico e prevenção de doenças monogênicas, e a seguir para as doenças complexas, os métodos diagnósticos e de investigação baseados na biologia molecular vão se consolidando e chegam aos pequenos laboratórios do interior. Dos métodos relativamente simples de detecção de mutações no DNA, estamos evoluindo para abordagens que permitem identificar padrões diversos de expressão gênica, com aplicações diagnósticas, preventivas e para intervenções terapêuticas.

As abordagens de manipulação, separação e expansão ex vivo de diferentes células progenitoras obtidas de embriões de sangue de cordão umbilical e de adultos tornaram-se uma realidade que promete modificar muitas das práticas médicas em curto prazo. Muitos são métodos que estão ainda em fase experimental, mas a perspectiva de virem a ser tecnologias de ampla aplicação no futuro exige a participação ativa dos pesquisadores brasileiros para evitar uma exagerada dependência externa futura

Qual nossa posição neste panorama? Um conjunto de iniciativas da Fapesp - <http://www. fapesp.br>, do MCT e do CNPq articulou em curto tempo uma ampla qualificação de pesquisadores e a expansão da infra-estrutura de mais de uma centena de laboratórios no país para a pesquisa em genômica e biologia celular. Recente relatório de uma comissão de especialistas da Organização Mundial da Saúde reconhece a posição de destaque alcançada pelo Brasil neste campo (WHO, 2002). O sucesso alcançado por esses programas na área básica (Collins, 2000) está se transferindo para as aplicações clínicas: a utilização de métodos de biologia molecular para diagnóstico e prevenção de doenças já é rotineira em muitos centros. Iniciativas em curso envolvem o uso de numerosos marcadores moleculares como fatores de risco ou de prognóstico, avaliação de expressão gênica em doenças específicas e seu valor diagnóstico e prognóstico, manipulação de células para tratamento de câncer e transplantes para regeneração de tecidos destruídos ou constitutivamente anormais, organização de bancos de sangue de cordão umbilical, entre outros.

Alguns aspectos da genômica são reconhecidos como importantes para garantir a saúde dos países em desenvolvimento, incluindo (WHO, 2002): doenças hereditárias da hemoglobina, resistência inata a doenças infecciosas, o diagnóstico de doenças infecciosas, resistência a drogas de agentes infecciosos, farmacogenômica e as doenças não-infecciosas, em especial o câncer. As recomendações para seu de- 
senvolvimento contemplam a colaboração internacional e regional, fortalecimento dos instrumentos de comunicação (entre cientistas e com a população) e da bioinformática.

A consolidação desta área, transferindo para a clínica as aplicações da biologia molecular e celular, é indispensável não apenas para que a prática médica no país mantenha-se atualizada e competitiva, mas também para permitir as interações com o setor produtivo para implantar um parque biotecnológico nacional. A competência alcançada pelo país neste setor deve servir de base para o desenvolvimento de uma indústria biotecnológica de aplicação médica, em especial para produção de vacinas e outras proteínas de aplicação médica (como hormônios e fatores de coagulação).

\section{As relações entre a indústria farmacêutica e os centros de pesquisa médica}

Como já apontado, grande parte da pesquisa médica e biomédica do país se faz em cerca de duas dezenas de instituições quase que exclusivamente públicas vinculadas a universidades e em muitos casos a hospitais universitários. Uma série recente de três artigos na mais prestigiosa revista médica americana, The New England Journal of Medicine (Stelfox et al., 1998; Angell, 2000; Bodenheimer, 2000; Correspondence, 2000) chamou a atenção para um problema de importância crescente: as relações cada vez mais intranqüilas entre as instituições acadêmicas e a indústria farmacêutica e de biotecnologia.

Essas relações, no entanto, precisam ser esclarecidas e normalizadas, pois encontram-se no centro da questão da pesquisa médica aplicada. A indústria farmacêutica ocupa uma posição insubstituível no cenário médico atual: é ela quem põe à disposição de pacientes e médicos os medicamentos que, em última instância, consubstanciam a síntese de grande parte da pesquisa médica e biológica. Para assegurar a eficiência e segurança do produto a indústria deve promover testes em humanos. Além de vencer todas as etapas exigidas pelas agências reguladoras (em particular Food and Drug Adminstration - FDA, nos Estados Unidos) (Spilker, 2004) a indústria procura ganhar a confiança da classe médica e sobrepujar os concorrentes, por meio de publicações em revistas médicas e o envolvimento de figuras que têm visibilidade e podem, pela sua participação no processo, como autores, conferencistas, participantes de mesas-redondas e cursos de atualização, ajudar a convencer seus colegas a prescrever aquele medicamento.

Este é o ponto em que o interesse da indústria se superpõe aos interesses de parcela dos membros das instituições acadêmicas (especialmente médicos de departamentos clínicos e cirúrgicos, e de hospitais universitários), que controlam os serviços que dispõem de melhores condições de diagnóstico e seguimento de pacientes, e eram participantes privilegiados do processo de planejamento dos testes. Mais recentemente esta dependência da indústria farmacêutica em relação às instituições acadêmicas reduziu-se, uma vez que surgiram empresas médicas especializadas em testar medicamentos, quer no ambiente hospitalar como na comunidade. Também as empresas passaram a contratar especialistas que fazem todo o planejamento dos testes, restando aos participantes um papel operacional. Este tipo de evolução afastou ou quase extinguiu as avaliações independentes sobre medicamentos, planejadas e conduzidas exclusivamente pelas instituições acadêmicas, e assegurou um firme controle da indústria farmacêutica sobre esta atividade, na qual aplica anualmente cerca de US\$ 6 bilhões, controlando cerca de $70 \%$ de todos os investimentos em testes clínicos de medicamentos (Bodenheimer, 2000).

Há substancial evidência sobre o efeito deletério que esta interferência crescente da indústria tem sobre atividades que deveriam ter um perfil exclusivamente acadêmico (Angell, 2000; Bodenheimer, 2000), dos quais o mais importante é a perda de objetividade científica produzindo viés favorável aos interesses da indústria (Stelfox et al., 1998). As evidências são suficientes para sustentar a noção de que os desvios ocorrem, embora não de uma forma intencional, sendo as situações mais graves representadas por conflitos entre pesquisadores e as companhias (visando impedir a publicação de resultados desfavoráveis), a perda do controle sobre os dados, a edição e a publicação do trabalho.

Os efeitos sobre instituições universitárias são igualmente preocupantes: a) utilização da capacidade de pesquisa (infra-estrutura e pessoal) da universidade para atender a um interesse comercial; b) reforço do poder dos grupos com capacidade de testar medicamentos, independentemente de seu desempenho acadêmico; c) estímulo, por parte de administrado- 
res de universidades e hospitais, dessa nova forma de angariar recursos. Acresce que as companhias pagam de maneira irregular e pouco expressiva aos pesquisadores acadêmicos brasileiros, e estes recursos muito raramente são controlados pelas instituições e investidos no seu interesse. Uma conseqüência indesejável para ambas as partes é a crescente perda de credibilidade da comunidade médica com relação aos resultados destas pesquisas ou testes. Muito tem contribuído para isso a identificação dos vieses dos resultados de estudos apoiados pela indústria em comparação com os resultados de estudos não-subvencionados, e a revelação da hierarquia organizacional que preside seu planejamento, execução e divulgação, que inclui o convite a cientistas eminentes para associarem seu nome à divulgação do trabalho escrito por ghostwriters contratados pela indústria.

Finalmente há que se considerar um aspecto particular desta relação entre universidade e indústria no Brasil. O emprego da capacidade clínica brasileira para testes de medicamentos não contribuiu para reforçar a indústria nacional, divergindo de outras situações, em que a universidade tem (ou pode ter) um papel crítico para o desenvolvimento tecnológico e econômico do país, reforçando a indústria genuinamente nacional.

Certamente as instituições médicas e universitárias precisam reavaliar suas relações com a indústria, no sentido de atender ao interesse público. Consoante afirmam Martin e Kasper (2000), as escolas e professores de medicina estão em uma posição difícil: são os guardiões dos investimentos públicos em pesquisa biomédica e ao mesmo tempo estão na vanguarda dos progressos científicos que têm que ser traduzidos em benefícios públicos pela indústria. A pesquisa em que novos medicamentos, agentes biológicos ou equipamentos médicos são testados em pacientes deve ser conduzida de forma que não exista a possibilidade (ou mesmo a desconfiança) de que o julgamento do pesquisador seja obscurecido pela perspectiva de ganho financeiro.

Há no momento duas abordagens, complementares entre si, para reduzir os aspectos negativos apontados nesta relação: a revelação detalhada de todos os potenciais conflitos de interesse de cada pesquisador, e os comitês institucionais de controle. No primeiro caso, todos os autores de trabalhos publicados, comunicações em congressos, conferencistas em eventos médicos, deveriam enunciar todas as formas de vínculos financeiros com a indústria (isto in- clui, por exemplo, apoio a viagens e pró-labore). Recentemente os editores de 12 revistas médicas de alta projeção (Davidoff et al., 2001; Campbell, 2001) decidiram que quando autores submetem um manuscrito, seja um artigo ou carta, eles são responsáveis por revelar todas as relações financeiras e pessoais entre eles e terceiros que possam causar viés ao seu trabalho. Para evitar ambigüidade, os autores devem esclarecer explicitamente se existem ou se não existem potenciais conflitos de interesse. Os autores devem revelar os potenciais conflitos de interesse aos participantes dos estudos, e devem acrescentar ao manuscrito a informação se de fato fizeram isso. Nesta mesma linha, há uma tendência a incluir nos artigos que contemplam grandes estudos de teste de medicamentos, informações sobre a hierarquia de organização do trabalho (como, por exemplo, quem foi o responsável pelo planejamento do trial, quem se responsabilizou pela análise estatística global, quem redigiu o manuscrito).

Quanto à segunda abordagem, há ainda grande variação, mas muitas das grandes instituições norte-americanas dispõem de comissões e instituições (diferente das comissões de ética médica ou em pesquisa, que focalizam principalmente o respeito aos interesses dos pacientes) que monitoram o envolvimento de seus docentes e médicos com a indústria, e fixam diretrizes éticas, de conduta e de vantagens financeiras. Longe de representar um empecilho à participação da academia na pesquisa clínica de interesse da indústria, esta solução dá-lhe uma característica institucional, removendo o aspecto de interesse pessoal do médico ou docente, e garantindo atenção prioritária ao interesse público. Em alguns casos a negociação dos contratos de prestação de serviço se faz diretamente entre as instituições industriais e acadêmicas.

Em resumo, as inter-relações entre a indústria farmacêutica e a comunidade acadêmica são necessárias, podem ser benéficas para ambas as partes e atender ao interesse público, desde que respeitados limites éticos e códigos de conduta que protejam o processo contra desvios voluntários ou involuntários, estes últimos muito mais freqüentes. 


\section{Agradecimentos}

O presente texto é de responsabilidade exclusiva do seu autor. No entanto, os conceitos e opiniões expressas derivam de colaborações em trabalhos anteriores e discussões com outros pesquisadores, em particular Jair J. Mari, Protásio L. da Luz, José R. Carvalheiro, Eduardo Ferriolli, Júlio C. Moriguti e Dimas T. Covas.

\section{Referências bibliográficas}

Almeida-Filho $\mathrm{N}$ et al. 1992. Estudo multicêntrico de morbidade psiquiátrica em áreas urbanas brasileiras (Brasília, São Paulo, Porto Alegre). Revista ABP-APAL 14(3):93-104.

Almeida-Filho N et al. 1997. Brazilian multicentric study of psychiatric morbidity. British Journal of Psychiatry 171:524-529.

Angell M 2000. Is academic medicine for sale? New England Journal of Medicine 342:1516-1518.

Bodenheimer T 2000. Uneasy alliance. Clinical investigators and the pharmaceutical industry. New England Journal of Medicine 342:1539-1544.

Bonalume Neto R 1999. Brazilian scientists team up for cancer genome project. Nature 398:450.

Buss PM 2002. Genômica e saúde pública. Cadernos de Saúde Pública 18:554-555.

Campbell P 2001. Declaration of financial interests. $\mathrm{Na}$ ture 412:751.

Collins P 2000. Fruits of cooperation. The Economist, ed. 8180, July 22-29.

Correspondence 2000. Is academic medicine for sale. New England Journal of Medicine 343: 508-510. [Além dos três artigos originais, a leitura da correspondência provocada por um deles é tão ilustrativa quanto o próprio artigo].

Davidoff F et al. 2001. Sponsorship, authorship, and accountability. New England Journal of Medicine 345: 825-827.

Dias Neto E et al. 2000. Shotgun sequencing of the human transcriptome with ORF expressed sequence tags. Proceedings of the National Academy of Sciences of USA 97:3491-3496.

Instituto Nacional do Câncer 2001. Estimativas de incidência e mortalidade por câncer no Brasil. Ministério da Saúde, Brasil.

Mari JJ 1987. Morbilidad psiquiátrica en centros de atención primaria. Boletin de la Oficina Sanitaria Panamericana 104(2):171-181.

Mari JJ 1992. Desigualdade social e saúde mental no Brasil. Boletim de Psiquiatria 25:5-10.

Mari JJ \& Zago MA 2002. O Brasil no cenário científico. Tendência/Debates. Folha de S. Paulo, 31 de maio de 2002.

Martin JB \& Kasper DL 2000. In whose best interest? Breaching the academic-industrial wall. New England Journal of Medicine 343:1646-1649.
Murray CJL \& Lopez AD 1996. The Global Burden of Disease. A comprehensive assessment of mortality and disability from diseases, injuries, and risk factors in 1990 and projected to 2020. The Harvard School of Public Health, World Health Organization and The World Bank, Harvard University Press, Boston.

Ramos LR et al. 1987. Envelhecimento populacional: uma realidade brasileira. Revista de Saúde Publica 21:211224.

Souza SJ et al. 2000. Identification of human chromosome 22 transcribed sequences with ORF expressed sequence tags. Proceedings of the National Academy of Sciences of U S A 97:12690-12693.

Spilker BA 2004. The drug development and approval process. Disponível em $<$ http://www.phrma.org $>$.

Stelfox HT, Chua G, O’Rourke K \& Detsky AS 1998. Conflict of interest in the debate over calcium-channel antagonists. New England Journal of Medicine 338:101-106.

Tauil PL 2002. Critical aspects of dengue control in Brazil. Cadernos de Saúde Pública 18:867-871.

Weatheral DJ 1985. The new genetics and clinical practice. (2a ed.). Oxford University Press, Oxford.

WHO 2002. Genomics and world health. Report of the Advisory Committee on Health Research.

Zago MA 2001. Anemia falciforme e doenças falciformes. Manual de Doenças mais Importantes, por Razões Étnicas, na População Brasileira Afro-Descendente. Série A. Normas e Manuais Técnicos no. 123, Ministério da Saúde, Brasília.

Zago MA \& Costa FF 1985. Hereditary haemoglobin disorders in Brazil. Transactions of the Royal Society of Tropical Medicine and Hygiene 79:385-388.

Zago MA, Costa FF, Tone LG \& Bottura C 1983. Hereditary hemoglobin disorders in a Brazilian population. Human Heredity 33:125-129.

Zago MA, Mari JJ, Carvalheiro JR, Silva LJ \& Luz PL 2002. Área de ciências da saúde. Academia Brasileira de Ciências. Contribuições para a Conferência Nacional de Ciência, Tecnologia e Inovação. Parcerias Estratégicas (Edição Especial), vol. 4.

Artigo apresentado em 8/12/2003

Aprovado em 17/2/2004

Versão final apresentada em 2/3/2004 